\title{
The Clinical Predictors of Shunt-Dependent Hydrocephalus Following Aneurysmal Subarachnoid Hemorrhage
}

\author{
Sio long $\mathrm{CHANG}^{1,2}$, Ming Dar TSA ${ }^{3,5}$, David Hung-Tsang YEN ${ }^{1,4}$, Cheng-Ta HSIEH ${ }^{5,6}$ \\ ${ }^{1}$ National Yang-Ming University, College of Medicine, Institute of Emergency and Critical Care Medicine, Taipei, Taiwan \\ ${ }^{2}$ Yonghe Cardinal Tien Hospital, Department of Neurosurgery, New Taipei City, Taiwan \\ ${ }^{3}$ Shin Kong Wu-Ho-Su Memorial Hospital, Department of Neurosurgery, Taipei, Taiwan \\ ${ }^{4}$ Taipei Veterans General Hospital, Department of Emergency Medicine, Taipei, Taiwan \\ ${ }^{5}$ Fu-Jen Catholic University, School of Medicine, New Taipei City, Taiwan \\ ${ }^{6}$ Cathay General Hospital, Department of Surgery, Division of Neurosurgery, Taipei, Taiwan
}

\section{ABSTRACT}

AIM: Shunt-dependent hydrocephalus $(\mathrm{SDH})$ is a relatively common complication following aneurysmal subarachnoid hemorrhage $(\mathrm{aSAH})$. Delay in diagnosis and treatment may worsen neurological outcome. This study was conducted to identify early clinical factors associated with SDH following aSAH.

MATERIAL and METHODS: Patients diagnosed with aSAH at our hospital from January 2010 through July 2014 were included. Patients aged $\leq 18$ or $\geq 90$ years, with concurrent arteriovenous malformation, treated with both clipping and coiling, or not receiving definitive treatment were excluded. Both clinical and radiological variables were analyzed by univariate and multivariate logistic model to identify factors independently associated with outcome of SDH following aSAH.

RESULTS: Overall, 33 patients (36.2\%) developed SDH following aSAH after definitive treatment. Univariate analysis revealed age, emergency department (ED) white blood cell (WBC) count, hypernatremia, Hunt \& Hess Grade, modified Fisher's Grade, presentation of acute hydrocephalus, initial placement of external ventriculostomy, and post-operative central nervous system infection were clinically significant factors. Multivariate logistic regression showed ED WBC count $>14,500 / \mu \mathrm{L}(\mathrm{OR} 5.096,95 \% \mathrm{Cl}$ 1.332 to $19.499, \mathrm{p}=0.017$ ), poor Hunt\&Hess Grade (III, IV \& V) (OR 3.562, 95\% Cl 1.081 to 11.737, $\mathrm{p}=0.037$ ), and initial placement of external ventriculostomy (OR $4.294,95 \% \mathrm{Cl} 1.125$ to $16.393, p=0.033$ ) were independent factors.

CONCLUSION: Three early independent predictors of SDH were identified including poor Hunt\&Hess Grade, initial placement of external ventriculostomy and ED leukocytosis. Early placement of a ventricular-peritoneal shunt after aSAH may shorten length of stay in hospital and in intensive care unit.

KEYWORDS: Aneurysmal subarachnoid hemorrhage, Shunt-dependent hydrocephalus, Clinical predictor, Outcome

\section{INTRODUCTION}

$\mathrm{S}$ hunt-dependent hydrocephalus $(\mathrm{SDH})$ is one of the major complications of aneurysmal subarachnoid hemorrhage (aSAH). It accounts for $4.3 \%$ to $48 \%$ of complications $(7,8,19,23,24,31,33,38)$. The cause of SDH has not been well established but it differs from that of acute hydrocephalus $(4,7,38)$. Blood clot, fibrin, and protein products after acute bleeding into the subarachnoid space obstruct or inhibit the arachnoid villi have been posed to result in acute hydrocephalus after aSAH (7). However, these meningeal reactions induced by aSAH that leads to arachnoid inflammation and adhesion, which blocks cerebrospinal fluid (CSF) absorption at the arachnoid villi and basal cisterns may result into $\operatorname{SDH}(4,9,38)$. The actual cause of SDH may be multi-factorial etiologies and 
further evaluation of clinical conditions is needed for additional clarification (11).

The patients with SDH following aSAH present with significant cognitive dysfunction and neurological deficit (40). Early placement of a permanent ventricular-peritoneal (V-P) shunt has been posed (15), but it remains controversial due to major complications (10). Advanced age, poor admission Hunt\&Hess Grade, initial brain computed tomography (CT) findings, admission blood glucose, hypertension, treatment modality, initial external ventricular drainage (EVD), meningitis or vasospasm are risk factors for $\mathrm{SDH}$ following aSAH $(7,8,11,26,35)$. However, the early change of the intracranial condition after aSAH remains unclear in the development of $\mathrm{SDH}$. Understanding the early clinical characteristics that are related to the prediction of SDH after surgical intervention for aSAH would provide invaluable information for neurosurgeons to manage patients in their early presentations. To our knowledge, only a few studies have evaluated the early clinical predictors of SDH after aSAH (26). Therefore, the aim of this present study was to identify the early potential predictors of outcome in patients with SDH after aSAH. These early predictors may provide the indication of early V-P shunt procedure, improve the patient's neurological outcome, and also shorten intensive care unit (ICU) and hospital length of stay (LOS).

\section{MATERIAL and METHODS}

\section{Patient Selection}

All patients diagnosed as aSAH at Shin-Kong Memorial Hospital between January 1, 2010 and July 31, 2014 were retrospectively reviewed and enrolled in this study. The study was approved by the hospital's institutional review board (IRB no. 20150201R). Patients aged $\leq 18$ or $\geq 90$ years, having concurrent arteriovenous malformation (AVM), having undergone combined treatment with clipping and coiling, or not receiving definitive treatment were excluded (Figure 1).

The diagnosis of spontaneous SAH was made by CT scan in the emergency department (ED) with the findings of $\mathrm{SAH}$ in the basal cistern or sylvian fissure without major trauma. The patients then underwent CT-angiography (CTA) or digital subtraction angiography (DSA) to accurately identify the location of vascular lesions. Definitive treatment was defined as surgical clipping or intravascular coiling in aneurysm. The decision about the treatment choice was based on the clinical decisions of neurosurgeons, neuroradiologists and the preference of patients or their families.

\section{Clinical Variables}

The clinical characteristics of all the study patients including age, gender, initial mean arterial blood pressure at the ED, ED glucose, ED white blood cell (WBC) count, serum sodium, operation method (clipping or coiling), EVD, and duration of external ventriculostomy placement were all recorded. Post-operative complications such as vasospasm or central nervous system (CNS) infection were also documented. Initial neurological status was evaluated using Hunt\&Hess Grade (14).

\section{Radiological Variables}

Brain CT images taken at the ED were used to evaluate the amount of subarachnoid blood using modified Fisher's Grade (6), location of aneurysm by anterior circulation (including the anterior cerebral artery, anterior communicating artery, posterior communicating artery, internal carotid artery, middle cerebral artery) or posterior circulation (including the posterior cerebral artery, basilar artery, vertebral artery, posteriorinferior cerebellar artery, anterior-inferior cerebellar artery, superior cerebellar artery), presence of acute hydrocephalus, intraventricular hemorrhage, and intracerebral hemorrhage.

\section{Outcome Measurement}

Shunt-dependent hydrocephalus was defined as clinical symptoms of hydrocephalus (including symptoms of increase intracranial pressure, cognitive dysfunction, gait disturbance and/or urinary incontinence) and radiological evidence of ventriculomegaly with bi-caudate index $(\mathrm{Bl})>95^{\text {th }}$ percentile for age $(12,13)$ that requiring the insertion of a permanent $V-P$ shunt. LOS in both the ICU and hospital were also recorded. Modified Rankin Scale (mRS) score at hospital discharge and 6 months after hospital discharge were evaluated (32).

\section{Statistical Analysis}

The clinical characteristics and radiographic variables between patients with non-SDH (NSDH) and those with SDH were compared to identify early predictive factors. Statistical analysis was performed using SPSS software version 12.0 (SPSS Inc., Chicago, IL). Statistical tests were two-sided, and the significance level was set at $p<0.05$. Data are presented as mean \pm standard deviation (SD) for continuous variables and as number (\%) for categorical variables. The distribution of the data was assessed with the Kolmogorov-Smirnov test. Comparisons of numerical variables were performed using an unpaired t-test (parametric data) or Mann-Whitney U-test (non-parametric data). Comparisons of categorical variables were done by chi-square or Fisher's exact test. Variables with $p$ value $<0.1$ in univariate analysis were entered into a multivariate logistic regression model to identify independent outcome predictors of SDH following aSAH.

\section{RESULTS}

\section{Patient Characteristics}

A total of 91 patients who underwent definitive treatment for aSAH were included in this study. A flowchart of study patients is shown in Figure 1. Thirty-three (36.3\%) of these patients received a shunt operation, either a clipping or coiling operation, with a mean 26.5 days after onset of aSAH. Patients in the SDH group were significantly older than those in the NSDH group (61.0 vs. 54.9 years, $\mathrm{p}<0.05$ ) (Table I).

In univariate analysis, several clinical and radiological variables were statistically significantly different between the NSDH and SDH groups: (1) age ( $p=0.046)$; (2) ED WBC count; (3) poor Hunt\&Hess Grade (III, IV \&V) ( $p=0.001)$; (3) hypernatremia $(p=0.003) ;(5)$ poor modified Fisher's Grade (III \& IV) ( $p=0.005)$; (6) initial acute hydrocephalus $(p=0.045)$; (7) initial placement 


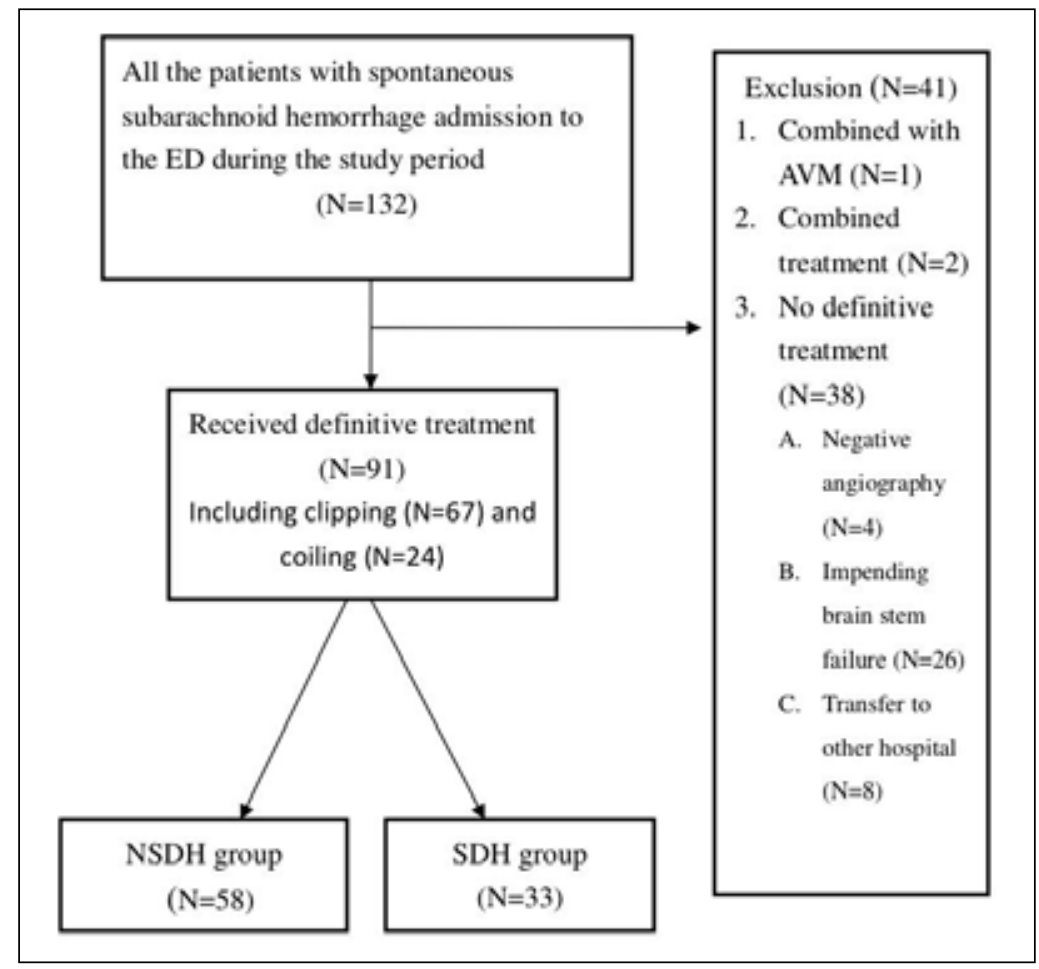

Figure 1: Flowchart of study patients. ED: Emergency department, SDH: Shunt-dependent hydrocephalus, NSDH: Non-shunt dependent hydrocephalus, AVM: Arteriovenous malformation. of EVD $(p=0.001)$, and (8) presence of CNS infection postoperatively $(p=0.015)$ (Table I). A cut-off value of ED WBC count at $14,500 / \mu \mathrm{L}$ demonstrated a greater statistically significant difference between the two groups. Also, patients who underwent external ventriculostomy of CSF drainage for more than 14 days had a higher odds ratio for development of SDH than those patients who did not undergo external ventriculostomy $(p=0.011)$.

\section{Outcome Measurements}

The mean ICU and hospital LOS for the SDH group were 24.1 days and 45.1 days, respectively. ICU and hospital LOS were significantly longer than for the NSDH group (14.2 days and 24.3 days, respectively) $(\mathrm{p}<0.001)$ (Table II). The mRS scores at discharge and at 6 months after discharge for the SDH patients were also poorer compared with the patients with NSDH (Table II). However, there was no significant difference in in-hospital mortality between the SDH and NSDH groups (Table II). The mean ICU (14.9 vs. 26.6 days) and hospital (25.3 vs. 50.5 days) LOS were significant longer if patients with $\mathrm{SDH}$ received operation more than 14 days following aSAH $(p=0.011$ and $p=0.005$, respectively). However, the $m R S$ score at discharge and 6 months were not significant $(p=0.349$ and $p=0.146$, respectively).

By applying multivariate logistic regression analysis, it was demonstrated that ED WBC count $>14,500 \mu \mathrm{L}$ (OR 5.096, $95 \% \mathrm{Cl} 1.332$ to $19.499, \mathrm{p}=0.017)$, poor Hunt\&Hess Grade (III, IV \&V) (OR 3.562, 95\% Cl 1.081 to $11.737, \mathrm{p}=0.037$ ), and initial placement of EVD (OR 4.294, 95\% Cl 1.125 to 16.393, $\mathrm{p}=0.033$ ) were clinical predictors associated with SDH postoperatively in patients who underwent surgery for aSAH (Table III).

\section{DISCUSSION}

SDH following aSAH remains a challenge for clinical physicians because of the unclear etiology. Early diagnosis is still the keystone in the management of SDH. However, the early clinical predictors of $\mathrm{SDH}$ after $\mathrm{aSAH}$ remain unclear and have been rarely discussed (26). In our present study, we identified the early potential predictors of outcome in patients and short-term outcome with SDH after aSAH. First, ED leukocytosis, poorer Hunt \& Hess grade at admission, and initial placement of EVD were significant clinical independent predictors of SDH following aSAH. Second, early placement of a $V-P$ shunt within 14 days resulted in shorter ICU and hospital LOS. Third, SDH patients had worse hospital discharge and 6-month post-discharge outcomes, and also longer ICU and hospital LOS.

It is well known that SAH induces a non-infectious acute systemic inflammatory response syndrome (SIRS) reaction, which is characterized by an increase in circulating cytokines and results in leukocytosis (36). Early elevation of CSF interleukin-6 (IL-6) has been described to significantly predict SDH (34). The relationship between leukocytosis and CSF cytokines after aSAH is unclear, but inflammation is reported to be related to other intracranial insults following aSAH $(21,22,27)$. Leukocytosis is also closely related to spontaneous intracerebral hemorrhage which affected early neurological deterioration (29). A recent study by Behrouz et al. (3) showed that admission leukocytosis has an inverse relationship with Glasgow Coma Scale (GCS) score but is not correlated with outcome. McMahon et al. (21) found that leukocytosis and change in IL-6 reflect impending cerebral ischemia. The mechanism explaining the relationship between early stage 
Table I: Patient Characteristics and Comparison of Clinical Variables Between Patients with and without Shunt-Dependent Hydrocephalus

\begin{tabular}{|c|c|c|c|c|}
\hline Patient characteristics & Total $(n=91)$ & NSDH $(n=58)$ & SDH (n=33) & p value \\
\hline \multicolumn{5}{|l|}{ Clinical variables } \\
\hline Age (years) ${ }^{*}$ & $57.1 \pm 14.2$ & $54.9 \pm 12.5$ & $61.0 \pm 16.3$ & 0.046 \\
\hline Female & $54(59.3)$ & $36(62.1)$ & $18(54.5)$ & 0.482 \\
\hline Mean ED MAP $(\mathrm{mmHg})$ & $115.5 \pm 19.6$ & $113.6 \pm 16.4$ & $118.8 \pm 24.5$ & 0.232 \\
\hline ED glucose (mg/dL) & $155.6 \pm 61.3$ & $158.2 \pm 67.0$ & $151.8 \pm 53.1$ & 0.693 \\
\hline ED WBC $>14.5\left(10^{3} / \mu \mathrm{L}\right)^{*}$ & $17(18.7)$ & $6(10.3 \%)$ & $11(33.3)$ & 0.007 \\
\hline ED WBC $\left(10^{3} / \mu \mathrm{L}\right)^{*}$ & $11.8 \pm 4.6$ & $11.0 \pm 3.8$ & $13.4 \pm 5.5$ & 0.030 \\
\hline Hyponatremia $(<135 \mathrm{mEq} / \mathrm{dL})^{*}$ & $21(23.1)$ & $13(22.4)$ & $8(24.2)$ & 0.842 \\
\hline Hypernatremia (>145 mEq/dL)* & $34(37.4)$ & $15(25.9)$ & $19(57.6)$ & 0.003 \\
\hline Hunt\&Hess Grade* & & & & 0.001 \\
\hline $\mathrm{I} / \mathrm{II}$ & $43(47.3)$ & $35(60.3)$ & $8(24.2)$ & \\
\hline III/IV/N & $48(52.7)$ & $23(39.7)$ & $25(75.8)$ & \\
\hline \multicolumn{5}{|l|}{ Operation variables } \\
\hline Initial EVD* & $57(62.6)$ & $29(50)$ & $28(84.8)$ & 0.001 \\
\hline EVD duration (days) $^{*}$ & $14.7 \pm 7.7$ & $12.3 \pm 7.3$ & $17.3 \pm 7.4$ & 0.013 \\
\hline Treatment method & & & & 0.728 \\
\hline Clipping & $67(73.6)$ & $42(72.4)$ & $25(75.8)$ & \\
\hline Coiling & $24(26.4)$ & $16(27.6)$ & 8 (24.2) & \\
\hline
\end{tabular}

\section{Radiologic variables}

\begin{tabular}{|c|c|c|c|c|}
\hline Modified Fisher's Grade* & & & & 0.005 \\
\hline $\mathrm{I} / \mathrm{II}$ & $24(26.4)$ & $21(36.2)$ & $3(9.1)$ & \\
\hline III/IV & $67(73.6)$ & $37(63.8)$ & $30(90.9)$ & \\
\hline Acute hydrocephalus* & $32(35.2)$ & $16(27.6)$ & $16(48.5)$ & 0.045 \\
\hline IVH & $41(45.1)$ & $23(39.7)$ & $18(54.4)$ & 0.170 \\
\hline $\mathrm{ICH}$ & $22(24.2)$ & $11(19.0)$ & $11(33.3)$ & 0.124 \\
\hline Aneurysm location & & & & 0.676 \\
\hline Anterior & $79(86.8)$ & $51(87.9)$ & $28(84.8)$ & \\
\hline Posterior & $12(13.2)$ & $7(12.1)$ & $5(15.2)$ & \\
\hline \multicolumn{5}{|l|}{ Related complications } \\
\hline CNS infection* & $4(4.4)$ & $0(0 \%)$ & $4(12.1)$ & 0.015 \\
\hline Clinical vasospasm & $22(24.2)$ & $13(22.4)$ & $9(27.3)$ & 0.603 \\
\hline
\end{tabular}

Results expressed as number (\%) for categorical variables and mean $( \pm S D)$ for numerical variables

NSDH: non-shunt dependent hydrocephalus, SDH shunt dependent hydrocephalus, ED: emergency department, MAP: mean arterial pressure, WBC: white blood cell, EVD: external ventricular drainage, IVH: intraventricular hemorrhage, ICH: intracerebral hemorrhage, CNS: central nervous system ${ }^{*} p<0.05$ means statistical significance in Mann-Whitney $U$ test or Chi-square or Fisher's exact test. 
Table II: Comparison of Outcomes Between NSDH and SDH Patients After aSAH

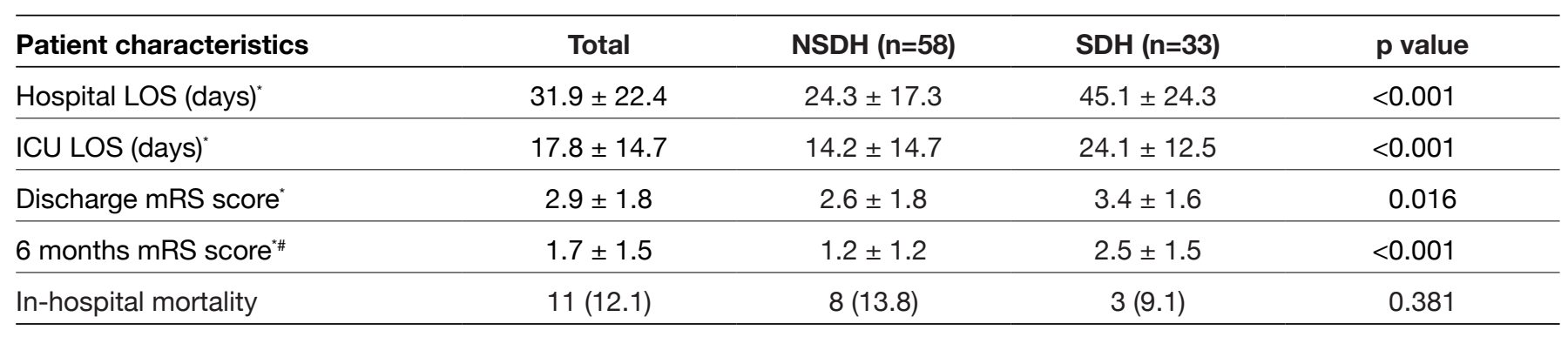

Results expressed as number (\%) for categorical variables and mean ( $\pm S D$ ) for numerical variables

aSAH: aneurysmal subarachnoid hemorrhage, NSD: non-shunt dependent hydrocephalus, SD: shunt dependent hydrocephalus,

LOS: length of stay, ICU: intensive care unit, $\mathbf{m R S}$ : modified Rankin Scale

${ }^{*} p<0.05$ means statistical significance in Mann-Whitney $U$ test or Chi-square or Fisher's exact test

\#11 patients lost to follow-up in NSDH group and 6 patients lost to follow-up in SDH group.

Table III: Independent Predictors of Shunt-Dependent Hydrocephalus After Asah by Multiple Regression Model

\begin{tabular}{lll}
\hline Factors & Odds ratio (95\% CI) & p value \\
\hline ED WBC $>14,500\left(10^{3} / \mu \mathrm{L}\right)^{*}$ & $5.096(1.332-19.499)$ & 0.017 \\
\hline EVD $^{*}$ & $4.294(1.125-16.393)$ & 0.033 \\
\hline Hunt\&Hess Grade III to V* & $3.562(1.081-11.737)$ & 0.037 \\
\hline Modified Fisher's Grade III/IV & $4.141(0.869-19.738)$ & 0.075 \\
\hline Age & $1.023(0.980-1.069)$ & 0.300 \\
\hline
\end{tabular}

aSAH: aneurysmal subarachnoid hemorrhage, ED: emergency department, WBC: white blood cell, EVD: external ventricular drainage,

" $p<0.05$ means statistical significance in regression analysis.

leukocytosis, aSAH and SDH is still unknown. In our series, we found leukocytosis detected in the early stage of aSAH had a strong relationship to SDH and outcomes. We believe that acute bleeding into the subarachnoid space induced inflammation of arachnoid villi and blocked CSF absorption which resulted in adhesion and chronic obstruction of arachnoid villi and finally became shunt dependent. Admission leukocytosis has not been proposed to be a predictor of SDH following aSAH in the literatures. Further study of anti-inflammatory treatment for aneurysm rupture in the early stage may be needed. It may improve the clinical condition at admission, which may in turn improve the incidence of SDH and outcome.

Poor clinical Hunt\&Hess Grade is a very important independent predictor in previous studies $(8,18)$. A large retrospective study, Dorai et al. (8) found $32 \%$ of patients with poor Hunt\&Hess Grade (III, IV) required a permanent V-P shunt. In our series, $52.1 \%(25 / 48)$ of patients with poor Hunt\&Hess Grade (III to V) received a permanent shunt procedure. Some previous studies excluded Hunt\&Hess Grade V patients due to the fact that the majority of such patients may not survive long enough to receive a V-P shunt $(8,18)$. After exclusion of 4 patients with Grade V, there were still 47.7\% (21/44) of our patients who received a permanent shunt procedure. Many factors may influence Hunt\&Hess Grade temporarily, including intraventricular hemorrhage (IVH) and acute hydrocephalus. Aggressive resuscitation and external CSF drainage initially may improve clinical grading and prolong survival of these kinds of patients. Initial EVD of CSF intermittently has been described as an independent predictor of SDH (35). In our study that longer duration (more than 2 weeks) of external CSF drainage revealed a significant relationship to $\operatorname{SDH}(p=0.011)$ in univariate analysis, which was compatible with those of previous studies $(1,13,28,29,35,39)$. Intermittent drainage of CSF can prevent the formation of occlusive membranes or clots $(1,13)$. Other CSF drainage methods such as lumbar drain (30) or lamina terminalis fenestration have been introduced to manage the initial aSAH (17). The relationship between lamina terminalis fenestration and SDH has been shown to be not significant (16). In addition, Bae et al. (2) found that a continuous lumbar drainage can reduce $\mathrm{SDH}$. Moreover, continuous external drainage of CSF may result in normal CSF pathways being occluded by blood clot that may lead to SDH. But, there still has been no extensive study or systematic review of continuous and intermittent drainage of CSF in SDH after aSAH.

Abnormal serum sodium is seldom discussed as a clinical predictor of SDH after aSAH. Appearance of abnormal serum sodium after spontaneous $\mathrm{SAH}$ is reported in the literature and hypernatremia is also a poor prognostic factor $(20,25)$. Yoshino et al. (37) postulated that mechanical pressure on the hypothalamus from $3^{\text {rd }}$ ventricle may have induced SIADH and resulting in hyponatremia. Li et al. (20) found 
negative correlation between serum sodium levels and cerebral ventricle size in SAH patients. However, we found no relationship between hyponatremia and SDH. A significant association was found between hypernatremia and SDH but this association was not found in multivariate analysis.

Early placement of a permanent $\mathrm{V}-\mathrm{P}$ shunt is still controversial due to major complications including infection and obstruction after the operation (10). Kang et al. (15) agree that early placement of permanent $\mathrm{V}-\mathrm{P}$ shunt after aSAH resulted in no shunt-related infection and low revision rate especially in those patients with high Fisher's Grade. In our study, early placement of a permanent V-P shunt showed shortening of both ICU and hospital LOS. These patients have a good initial performance and family support. However, there was no improvement of discharge or 6-month outcomes according to mRS scores.

The relationship between treatment method and shunt dependency is still controversial $(2,6)$. Endovascular coiling has been shown to increase the risk of shunt dependency after aSAH $(8,39)$. However, Zaidi et al. (40) revealed no difference of shunt dependency among patients by clip or coil. In our study, no significant difference between treatment method and $\mathrm{SDH}$ was found. Our study also revealed the radiographic variables, such as aneurysm location, IVH or ICH were not significant associated with SDH (Table I). In univariate analysis, a significant association was found between modified Fisher's Grade and acute hydrocephalus but this association was not found in multivariate analysis. These findings may have differed from those of previous studies because our patients had a predominance of advanced modified Fisher's Grade (III/ IV: $73.6 \%$ ). In this study, patients found to have IVH and acute hydrocephalus usually receive placement of EVD initially to remove IVH (88\%) and release acute hydrocephalus (81\%). This high percentage of patients who receive early intervention for acute hydrocephalus or IVH may decrease the influence of these brain CT variables in SDH.

Post-operative complications such as nosocomial meningitis and vasospasm have been found to be associated with $\operatorname{SDH}(13,29)$. In this study, it was found that CNS infection was significantly related to $\mathrm{SDH}$ in univariate analysis. Four patients had CNS infection and this may have resulted in $\mathrm{SDH}$. Their cultures yielded Peptostreptococcus spp, Gram positive cocci, Gram negative bacilli, and Viridans Streptococcus, respectively. Due to small patient numbers, this predictor was not included in multivariate regression analysis. Dorai et al. (8) described the significant correlation between vasospasm, cerebral ischemia, and SDH. In the present study, only $24.2 \%$ $(22 / 91)$ of patients were recorded to have symptomatic vasospasm. Subsequent transcranial Doppler (TCD) was not used routinely in our clinical practice; therefore, there may have been an underestimate of the incidence in our present study. Clinical silent cerebral vasospasm or silent cerebral ischemia may be missed. The use of TCD routinely to diagnose clinical silent vasospasm is necessary in further investigation.

The current study has several limitations. First, this is a retrospective study and some confounding variables may have not been measured and not collected. Second, hydrocephalus may develop in late stage after definitive treatment. We did not use follow-up brain CT regularly in all the treated patients, and also patients with poor outcome (mRS score $\geq 4$ ) was treated negatively. This may have resulted in an underestimation of the actual frequency of hydrocephalus. A prospective study in the future may be needed to assess the actual incidence and predictors of SDH following aSAH.

\section{- CONCLUSION}

Poor Hunt\&Hess Grade, ED leukocytosis, and initial placement of external ventriculostomy are identified as the independent predictors in patients with SDH following aSAH. Early prediction of SDH in patients after aSAH may help neurosurgeons decide whether to use early placement of a permanent V-P shunt. The results may provide additional information for neurosurgeons to improve neurological outcomes and shorten both ICU and hospital LOS for patients with aSAH.

\section{- REFERENCES}

1. Auer LM, Mokry M: Disturbed cerebrospinal fluid circulation after subarachnoid hemorrhage and acute aneurysm surgery. Neurosurgery 26: 804-809, 1990

2. Bae IS, Yi HJ, Choi KS, Chun HJ: Comparison of incidence and risk factors for shunt-dependent hydrocephalus in aneurysmal subarachnoid hemorrhage patients. J Cerebrovasc Endovasc Neurosurg 16(2):78-84, 2014

3. Behrouz R, Hafeez S, Miller CM: Admission leukocytosis in intracerebral hemorrhage: Associated factors and prognostic implications. Neurocrit Care 23(3):370-373, 2015

4. Blasberg R, Johnson D, Fenstermacher J: Absorption resistance of cerebrospinal fluid after subarachnoid hemorrhage in the monkey; Effects of heparin. Neurosurgery 9:686-691, 1981

5. Claassen J, Bernardini GL, Kreiter K, Bates J, Du YE, Copeland D, Connolly ES, Mayer SA: Effect of cisternal and ventricular blood on risk of delayed cerebral ischemia after subarachnoid hemorrhage: The Fisher scale revisited. Stroke 32:2012-2020, 2001

6. Dehdashti AR, Rilliet B, Rufenacht DA, de Tribolet N: Shuntdependent hydrocephalus after rupture of intracranial aneurysms: A prospective study of the influence of treatment modality. J Neurosurg 101(3): 402-407, 2004

7. Demirgil BT, Tugcu B, Postalci L, Guclu G, Dalgic A, Oral Z: Factors leading to hydrocephalus after aneurysmal subarachnoid hemorrhage. Minim Invasive Neurosurg 46(6):344-348, 2003

8. Dorai Z, Hynan LS, Kopitnik TA, Samson D: Factors related to hydrocephalus after aneurysmal subarachnoid hemorrhage. Neurosurgery 52(4):763-771, 2003

9. Ellington E, Margolis G: Block of arachnoid villus by subarachnoid hemorrhage. J Neurosurg 30: 651-657, 1969

10. Erdincler P, Kaynak MY, Canbaz B, Etus V, Dirican A, Ciplak N, Kuday C: Spreading of ventriculo-peritoneal shunt complications over time: An analysis of 210 patients and 388 procedures covering a period of seven years. Turk Neurosurg 8: $1-5,1998$ 
11. Erixon HO, Sorteberg A, Sorteberg W, Eide PK: Predictors of shunt dependency after aneurysmal subarachnoid hemorrhage: Results of a single-center clinical trial. Acta Neurochir (Wien) 156(11): 2059-2069, 2014

12. Haug G: Age and sex dependence of the size of normal ventricles on computed tomography. Neuroradiology 14: 201204, 1977

13. Hirashima $\mathrm{Y}$, Hamada $\mathrm{H}$, Hayashi N, Kuwayama N, Origasa $H$, Endo S: Independent predictors of late hydrocephalus in patients with aneurysmal subarachnoid hemorrhage-analysis by multivariate logistic regression model. Cerebrovasc Dis 16: 205-210, 2003

14. Hunt WE, Hess RM: Surgical risk as related to time of intervention in the repair of intracranial aneurysms. J Neurosurg 28(1):14-20, 1968

15. Kang DH, Park J, Park SH, Kim YS, Hwang SK, Hamm IS: Early ventriculoperitoneal shunt placement after severe aneurysmal subarachnoid hemorrhage: Role of intraventricular hemorrhage and shunt function. Neurosurgery 66(5): 904-909, 2010

16. Komotar RJ, Hahn DK, Kim GH, Starke RM, Garrett MC, Merkow MB, Otten ML, Sciacca RR, Connolly ES Jr: Efficacy of lamina terminalis fenestration in reducing shunt-dependent hydrocephalus following aneurysmal subarachnoid hemorrhage: A systematic review. J Neurosurg 111(1):147-154, 2009

17. Komotar RJ, Olivi A, Rigamonti D, Tamargo RJ: Microsurgical fenestration of the lamina terminalis reduces the incidence of shunt-dependent hydrocephalus after aneurysmal subarachnoid hemorrhage. Neurosurgery 51(6):1403-1413, 2002

18. Lai L, Morgan MK: Predictors of in-hospital shunt-dependent hydrocephalus following rupture of cerebral aneurysms. J Clin Neurosci 20(8):1134-1138, 2013

19. Lanzino G, Kassell NF, Germanson TP, Kongable GL, Truskowski LL, Torner JC, Jane JA: Age and outcome after aneurysmal subarachnoid hemorrhage: Why do older patients fare worse? J Neurosurg 85: 410-418, 1996

20. Li M, Li W, Wang L, Hu Y, Chen G: Relationship between serum sodium level and brain ventricle size after aneurysmal subarachnoid hemorrhage. Acta Neurochir Suppl 105: 229232, 2008

21. McMahon CJ, Hopkins S, Vail A, King AT, Smith D, Illingworth KJ, Clark S, Rothwell NJ, Tyrrell PJ: Inflammation as a predictor for delayed cerebral ischemia after aneurysmal subarachnoid haemorrhage. J Neurointerv Surg 5(6): 512-517, 2013

22. Miller BA, Turan N, Chau M, Pradilla G: Inflammation, vasospasm, and brain injury after subarachnoid hemorrhage. Biomed Res Int 2014:384342, 2014

23. O'Kelly CJ, Kulkarni AV, Austin PC, Urbach D, Wallace MC: Shunt-dependent hydrocephalus after aneurysmal subarachnoid hemorrhage: Incidence, predictors, and revision rates. Clinical article. J Neurosurg 111: 1029-1035, 2009

24. Pietilä TA, Heimberger KC, Palleske H, Brock M: Influence of aneurysm location on the development of chronic hydrocephalus following SAH. Acta Neurochir (Wien) 137: 7073, 1995

25. Qureshi Al, Suri MF, Sung GY, Straw RN, Yahia AM, Saad M, Guterman LR, Hopkins LN: Prognostic significance of hypernatremia and hyponatremia among patients with aneurysmal subarachnoid hemorrhage. Neurosurgery 50(4): 749-755, 2002
26. Rincon F, Gordon E, Starke RM, Buitrago MM, Fernandez A, Schmidt JM, Claassen J, Wartenberg KE, Frontera J, Seder DB, Palestrant D, Connolly ES, Lee K, Mayer SA, Badjatia N: Predictors of long-term shunt-dependent hydrocephalus after aneurysmal subarachnoid hemorrhage. J Neurosurg 113(4): 774-780, 2010

27. Spallone A, Acqui M, Pastore FS, Guidetti B: Relationship between leukocytosis and ischemic complications following aneurysmal subarachnoid hemorrhage. Surg Neurol 27(3): 253-258, 1987

28. Sugawara T, Maehara T, Tadashi N, Aoyagi M, Ohno K: Independent predictors of shunt-dependent normal pressure hydrocephalus after aneurysmal subarachnoid hemorrhage. $J$ Neurosurg Sci 60(2):154-158, 2016

29. Sun C, Du H, Yin L, He M, Tian Y, Li H: Choice for the removal of bloody cerebrospinal fluid in postcoiling aneurysmal subarachnoid hemorrhage: External ventricular drainage or lumbar drainage? Turk Neurosurg 24(5):737-744, 2014

30. Sun W, Peacock A, Becker J, Phillips-Bute B, Laskowitz DT, James ML: Correlation of leukocytosis with early neurological deterioration following supratentorial intracerebral hemorrhage. J Clin Neurosci 19(8): 1096-1100, 2012

31. Tapaninaho A, Hernesniemi J, Vapalahti M, Niskanen M, Kari A, Luukkonen M, Puranen M: Shunt-dependent hydrocephalus after subarachnoid haemorrhage and aneurysm surgery: Timing of surgery is not a risk factor. Acta Neurochir (Wien) 123: 118-124, 1993

32. van Swieten JC, Koudstaal PJ, Visser MC, Schouten HJ, van Gijn J: Interobserver agreement for the assessment of handicap in stroke patients. Stroke 19: 604-607, 1988

33. Vermeij FH, Hasan D, Vermeulen M, Tanghe HL, van Gijn J: Predictive factors for deterioration from hydrocephalus after subarachnoid hemorrhage. Neurology 44: 1851-1855, 1994

34. Wostrack M, Reeb T, Martin J, KehI V, Shiban E, Preuss A, Ringel F, Meyer B, Ryang YM: Shunt-dependent hydrocephalus after aneurysmal subarachnoid hemorrhage: The role of intrathecal interleukin-6. Neurocrit Care 21(1): 78-84, 2014

35. Yang TC, Chang CH, Liu YT, Chen YL, Tu PH, Chen HC: Predictors of shunt-dependent chronic hydrocephalus after aneurysmal subarachnoid haemorrhage. Eur Neurol 69: 296303, 2013

36. Yoshimoto Y, Tanaka Y, Hoya K: Acute systemic inflammatory response syndrome in subarachnoid hemorrhage. Stroke 32(9): 1989-1993, 2001

37. Yoshino M, Yoshimi Y, Taniguchi M, Nakamura S, Ikeda T: Syndrome of inappropriate secretion of antidiuretic hormone associated with idiopathic normal pressure hydrocephalus. Intern Med 38(3): 290-292, 1999

38. Yoshioka H, Inagawa T, Tokuda Y, Inokuchi F: Chronic hydrocephalus in elderly patients following subarachnoid hemorrhage. Surg Neurol 53: 119-125, 2000

39. Yu H, Zhan R, Wen L, Shen J, Fan Z: The relationship between risk factors and prognostic factors in patients with shuntdependent hydrocephalus after aneurysmal subarachnoid hemorrhage. J Craniofac Surg 25(3): 902-906, 2014

40. Zaidi HA, Montoure A, Elhadi A, Nakaji P, McDougall CG, Albuquerque FC, Spetzler RF, Zabramski JM: Long-term functional outcomes and predictors of shunt-dependent hydrocephalus after treatment of ruptured intracranial aneurysms in the BRAT trial: Revisiting the clip vs coil debate. Neurosurgery 76(5): 608-614, 2015 